\title{
Placental pathology in low birth weight babies a prospective observational study
}

\author{
Chaithra R Jadhav ${ }^{1}$, Banushree C Srinivasamurthy ${ }^{2, *}$, Ramchandra V Bhat ${ }^{3}$, Vinita Agrawal ${ }^{4}$, \\ Harendra Kumar ${ }^{5}$ \\ ${ }^{1,2}$ Associate Professor, ${ }^{3,5}$ Professor and HOD, ${ }^{4}$ Assistant Professor, ${ }^{1-4}$ Indira Gandhi Medical College and Research Institute, \\ Puducherry, ${ }^{5}$ Sri Devraj Urs Medical College, Kolar, Karnataka, India
}

*Corresponding Author:

Email: drbanushree15@hotmail.com

Received: $6^{\text {th }}$ November, 2017

Accepted: $27^{\text {th }}$ March, 2018

\begin{abstract}
Introduction: Low birth weight (LBW) babies are more common in developing countries like India. The placenta is an organ which connects fetus to mother during intrauterine life. Any change in the mother reflects on placenta and thus on the fetus.

Materials and Methods: In this study placenta from LBW were evaluated. We included 50 fresh placentae. Gross examination including weight, shape, thickness, feto-placental ratio was calculated. Later, histo-morphological study of all placentae was done. The extent of placental lesions which can influence the birth weight of the baby or adverse fetal outcome were correlated.

Results: Out of 50 placentae studied, there was significant reduction in placental weight and dimension. Irregular shape of placenta was seen in 13 cases and most of the plscenta (27 cases) showed paracentral insertion of umbilical cord. On gross, infarction [56\%], subchorionic fibrin [64\%], intervillous fibrin [52\%] were noted. On microscopy, cytotophoblast hyperplasia [52\%], stomal fibrosis [48\%], fibrinoid necrosis [100\%], synnctial knots [32-68\%], hypovascular villi [44\%] were noted.

Conclusion: All major gross and microscopic changes in the low birth weight placentas pointed towards reduced blood flow to the placenta resulting in chronic placental insufficiency. The severity of placental abnormalities expressed as cumulative number of placental lesions is a significant risk factor for LBW babies. Morphological examination of the placenta conducted in the present study proved to be a useful adjunct to the clinical examination in finding the pathogenetic mechanisms during pregnancy resulting in LBW babies and can be helpful in the planning and management of future pregnancies.
\end{abstract}

Keywords: Placental examination, Low birth weight babies, Pregnancy, Feto-placental ratio.

\section{Introduction}

In recent years a child with low birth weight [LBW] with or without signs of intrauterine malformation has attracted much attention. ${ }^{1}$ Fetal growth retardation is important because of its associated morbidity and mortality. ${ }^{2}$ The placenta as an ephemeral organ interposed between mother and fetus often is the target of insult directed at the fetus. It thus becomes dairy of gestation. ${ }^{3}$ Maternal factors contributing to LBW are anemia, nutritional deficiencies, genetic factor, congenital/ acquired heart disease, narcotic addiction, alcoholism. Fetal factors contributing to LBW are genetic factor, chronic infection, multiple pregnancies. ${ }^{1}$ All these causative factors are so closely linked that, it is not possible to implicate any factor alone as being causative in nature. Development of LBW, depends on duration and the time of placental insult and gestational period. ${ }^{4}$ Premature aging of the placenta is thought to be responsible for poor fetal outcome in LBW babies, in cases not associated with any maternal or fetal disorders. ${ }^{5}$ placental changes leading to retarded growth are infarct, asynchronous maturation of chronic villi, numerous syncitio capillary membranes, syncytial knots, massive perivillous fibrinoid changes and thick basement membrane. All these related to impaired circulation of intervillous space. ${ }^{6-8}$ Placenta depicts the most accurate record of prenatal experience of an infant. It undergoes different changes in weight, volume, structure, shape and function continuously throughout the gestation to support the prenatal life. ${ }^{9}$ The careful examination of placenta can reveal many facts that may either directly or indirectly explain the past event that potentially determine the outcome of pregnancy. ${ }^{3}$ There are various factors to be studied in villious abnormalities of placenta like cytotrophoblast proliferation, intervillous fibrin deposition, infarction, syncytial knots, stromal fibrosis, fibrinoid necrosis. Singleton pregnancies with marginal and velamentous placental cord insertion identified at the time of midtrimester anatomical survey are at increased risk of delivering small-for-gestational-age newborns. ${ }^{10}$

\section{Materials and Methods}

A total of fifty freshly delivered placentae were collected from the Department of Obstetrics and Gynaecology. Before the study, permission was taken from the Institutional Ethics Committee and Head of Obstetrics and Gynaecology. In each case, a preliminary history was elicited from the mother regarding her age, parity, the period of amenorrhoea, history of bleeding per vaginum and her present and previous obstetric history with regard to PIH and diabetes mellitus. Placentae of mothers who have delivered a low birth weight baby less than $2.5 \mathrm{kgs}$ were collected. The babies whose placentae were 
utilized in this study were examined for any visible anomalies and sex, weight, maturity of the baby was noted. All the placentae were collected immediately after delivery. The collected placenta was washed under running tap water. The cord and membranes were examined for any abnormalities. The placenta along with the cord were collected in $10 \%$ formalin filled wide mouthed plastic containers. All the specimens were tagged with number discs before the commencement of the study for the purpose of identification. Weight was measured using electronic weighing machine.

In the collected placenta the following parameters were studied on gross examination:

1. Weight: Measured using electronic weighing machine.

2. Shape: The shape of the placenta and the presence of accessory lobe were recorded after proper inspection. Each placenta was categorised round, oval or irregular in shape.

3. Diameter: The placenta was placed in a flat tray after trimming. At first, the maximum diameter was measured with a metallic scale graduated in centimetres. Then the second maximum diameter was taken at right angles to the first one. The mean of the two measurements is considered to be the diameter of the placenta expressed in centimetres.

4. Cord: length, number of umbilical vessels, presence of true knots, torsion, stricture, hematoma or thrombosis were recorded.

5. Number of Cotyledons: Each formalin fixed placenta was taken on both the hands. Then gentle pressure was applied on the central part of the foetal surface with thumbs of both the hands while holding the periphery of the placenta with the other fingers. As a result, the cotyledons on the maternal aspect became prominent after separation between them. Then the placenta was put in a flat tray with the maternal surface facing upwards by placing a block of paraffin on the foetal side. Then the counting was started from the left side of one end of the placenta going rightward and again turning back to the left in a manner of loop. This counting procedure was repeated until the other end of the placenta was reached. The total numbers of cotyledons were recorded.

6. The foeto-placental ratio

7. The colour of the placental membranes

8. The presence of sub chorionic fibrosis.

The presence of the following abnormal placental characteristics were looked for

1. Placental calcification

2. Amniotic bands

3. Retro-placental lobes.

4. Accessory placental lobes.
Transverse cuts were made through the maternal surface at a distance of 1-2 $\mathrm{cm}$ in bread loaf manner and examined for pale areas. The placenta was grossed by swiss-roll technique. Bits were taken from the maternal surface, membranes, cords and from the representative areas. The tissues were then processed, embedded and block out with paraffin wax. Haematoxylin and eosin stains were used and the slides were examined under a light microscope.

The microscopic study of the placenta was carried out utilising a set of standard criteria for villous and intervillous lesions. For studying these criteria eight random microscopic fields were chosen and hundred villi were counted in each field and studied for the presence of the following criteria:

\subsection{Villous Lesions}

1. Syncytial knots $>30 \%$ in one field

2. Fibrinoid necrosis $>5 \%$ in one field

3. Placental infarction $>5 \%$ in one field

\subsection{Intervillous Space}

1. Perivillous fibroid deposition $>5 \%$ in one field

2. Presence of calcification.

\section{Results and Discussion}

The placenta is essential for optimal fetal growth as it provides nutrients to the fetus. ${ }^{10}$ Intra uterine growth retardation can be due to maternal, fetal, placental and unknown causes, but the basic pathophysiology is due to either reduced availability of nutrients in mother or its reduced transfer by the placenta to the fetus and may also be due to reduced utilization by the fetus. ${ }^{9}$ LBW more frequently show multiple type of lesion in their placenta. Evidence suggests that fetal growth retardation occurs due to more than single lesion in uteroplacenta or villous. It is more likely that accumulation of placental lesion for sufficient time interval leads to LBW babies. ${ }^{3}$ Optimal fetal growth will require adequate maternal and fetal circulation, sufficient quantities of circulating nutrients in the maternal blood and proper area of placental membrane that is capable of achieving satisfactory maternal fetal transfer. ${ }^{11}$ A total of 50 placentae delivered to babies weighing $<2.5 \mathrm{~kg}$ were studied. The maximum number of placenta were obtained from primigravida in the age group of 21-25 yrs. (Table 1,2)

Table 1: Age distribution

\begin{tabular}{|l|c|c|}
\hline Age group & No of cases & \% \\
\hline$<20 \mathrm{yr}$ & 5 & 10 \\
\hline $21-25$ & 27 & 54 \\
\hline $26-30$ & 15 & 30 \\
\hline$>30$ & 3 & 6 \\
\hline Total & 50 & 100 \\
\hline
\end{tabular}


Table 2: Parity

\begin{tabular}{|l|c|c|}
\hline Parity & No of cases & \% \\
\hline Multi & 24 & 48 \\
\hline Primi & 26 & 52 \\
\hline Total & 50 & 100 \\
\hline
\end{tabular}

Table 3: Weight of the babies

\begin{tabular}{|l|c|c|}
\hline Wt of baby (kg) & No of cases & \% \\
\hline$<1$ & 1 & 2 \\
\hline 1.5 & 2 & 4 \\
\hline $1.6-2$ & 26 & 52 \\
\hline $2.1-2.5$ & 21 & 42 \\
\hline Total & 50 & 100 \\
\hline
\end{tabular}

The placenta of babies weighing $1.6-2 \mathrm{~kg}$ was maximum in number, followed by $2.1-2.5 \mathrm{~kg}$. (Table 3 )

In fetal growth restriction (FGR), where a fetus fails to achieve its genetically pre-determined growth potential, placental weight and birth weight: Placental Weight ratio are often reduced which may indicate a placenta that fails to adapt its nutrient transfer capacity to compensate for its small size. ${ }^{10}$ The normal placental weight is 650 gms with water being $84 \% .{ }^{12}$ In the present study, placental weight was 300-400 gms and constituted $64 \%$ of all cases. In Study by Mardi K et al, placental weight was 300-400 gms and constituted 68\% of all cases and similar findings were seen in Panti et al study as well. [3.13] (Table 4) Majority of placentae were oval in shape (26 cases) followed by irregular in 13 cases and round in 11 cases. In the present study, marginal and paracentral attachment of umbilical cord was 58\% correlating with Prabhjot et al study in which it was around 52\%. ${ }^{14}$ Marginal and para central cord insertions are associated with increased placental thrombi in fetal vessel leading to LBW. ${ }^{15}$ Marginal insertion of cord was associated with an increased risk of birth weight less than the $10^{\text {th }}$ percentile when compared to controls in a study conducted by Amelia et al. ${ }^{16}$

Table 4: Weight of placentae

\begin{tabular}{|l|c|c|}
\hline $\begin{array}{l}\text { Weight of } \\
\text { placenta }(\mathbf{g m s})\end{array}$ & No of cases & \% \\
\hline$<300$ & 10 & 20 \\
\hline $300-400$ & 32 & 64 \\
\hline$>400$ & 8 & 16 \\
\hline Total & 50 & 100 \\
\hline
\end{tabular}

In our study, the cord length varied from $12-43 \mathrm{~cm}$. A short cord $(<32 \mathrm{~cm})$ is a cause of cord rupture and delaying in fetal delivery. A long cord $(72 \mathrm{~cm})$ increases the risk of true knots, cord compression forom fetal entanglement. ${ }^{17}$ In our study the true length of the umbilical cord was not recorded as the clamping and cutting of the cord largely depends on obstetricians and nurses who conduct delivery. In present study, the average surface area was $339 \mathrm{~m} 3$. The placental volume, surface area and no of cotyledons were reduced (average $201 \mathrm{~m}^{3}$ in weight less than $2000 \mathrm{~g}$ ) in lower socioeconomic group and showed significant relationship with calorie, protein intake and LBWB similar to Mehendel et al study. ${ }^{4,18}$ Marked increase of hypoplastic placentas will be found among small for date infants. ${ }^{19}$ Rayburn et al found similar findings in placenta of growth retarded fetus. ${ }^{20}$ The cord was normal in 35 cases followed by under coiled and hyper twisted in 2 cases. Hyper twisted cords are usually associated with obstruction to circulation. ${ }^{12,} 21$ The membrane was semi opaque in 25 cases followed by opaque in 14 cases and clear in 11 cases. In Scott et al study meconium staining, amnion nodosum and excessive scarring of placenta noted. ${ }^{22}$ But meconium staining was not noted in our study. The membrane insertion was marginal in $41(82 \%)$ cases and circummarginal in $9(18)$ cases. The circumvallate membrane insertion and velamentous cord insertion association with LBW babies was noted in other studies but not in present study. ${ }^{20,23}$ Maternal surface was ragged in 2(4\%) cases and complete in $48(96 \%)$ cases. Ragged placentae were associated with excessive infarction and intervillous fibrin deposition on microscopy. In present study, there was infarction $(56 \%)$, calcification $(10 \%)$, subchorionic fibrin $(64 \%)$, intervillous fibrin (52\%), retroplacental hematoma (2\%) and normal in 16\%. (Table 5) [Fig. 1, 2] There was significantly high percentage of infarction, subchorionic fibrin and intervillous fibrin in comparison with Mardi et al and Mehendale et al studies. The percentage of retroplacental haemorrhage was correlating with the above mention studies. ${ }^{3,4}$ However, extensive infarction and calcification was noted in the study conducted by Chandra $\mathrm{p}$ et al. ${ }^{24}$ All the gross placental measurements were significantly lower in LBW compared to controls in an univariate analysis. However, only the placental weight, and the largest and smallest distances from the point of insertion of the umbilical cord were highly associated with LBW in a stepwise logistic regression analysis done by Larysa et al. $^{25}$ Microscopically, Intervillous fibrin was seen in $64 \%$ of placenta in our study. However in Bane et al study all the placenta showed intervillous fibrin. ${ }^{7}$ Only $48 \%$ and $27.4 \%$ in Bazaz et al and Scott et al studies respectively. ${ }^{5,22}$ This massive perivillous fibrin causes chronic placental insufficiency and is detremintal to fetal survival and outcome. $^{7}$ The syncytial knots occurs due to reduced perfusion of villi and morphologically it is the reflection of decreased uteroplacental blood flow. ${ }^{3,4}$ In our study, it was seen in 45\% similar to Mirchandani et al and Bazaz et al studies. ${ }^{26,5}$ In Other study, Chandra et al syncytial knots were noted in $60-80 \%$ of placentae. ${ }^{24}$ In stromal fibrosis, increased villous fibrosis is associated with LBWB which can lead to reduction in the functioning of the villi causing placental insuffiency. ${ }^{27}$ Stromal fibrosis was seen in $48 \%$ in our study. It was more frequently seen in Mardi et al. study 
$(56 \%)$ and in some placentae in Bazaz $M$ et al. study $(22-26 \%)^{3,5}$ cytotrophoblastic hyperplasia and basement membrane thickening are interrelated. Cytotrophoblastic hyperplasia becomes prominent and numerous in an attempt to replace and repair damaged syncytiotrophoblast in the placental ischemia. Fibrinoid necrosis is also associated with basement membrane thickening. Basement membrane thickening was noted in $52 \%$ and fibrinoid necrosis was seen in $100 \%$ of placenta in our study. ${ }^{5}$ Mixture of lymphocytes and hisiocytes were common finding in chronic villitis and was seen in $4 \%$ in the present, but in studies conducted by Mardi $\mathrm{K}$ et al and Altemani AM et al villitis was seen in $12 \%$ and $32 \%$ of cases respectively. ${ }^{3,24,28}$ Studies have suggested an association between LBW and villitis of either hematogenous infection or villitis of unknown etiology. ${ }^{29}$ Nigam et al, On microscopic examination placental ischemia, infarction and calcification were seen in significantly higher number of patients than controls. Fibrinoid necrosis, stromal fibrosis, placental dysmaturity and obstructive vasculapathy were seen in placentae from patients only. ${ }^{9}$ [Fig. 3,4]. All the microscopic features found in our study is summarized in table 6 .

Table 5: Gross examination

\begin{tabular}{|l|c|c|c|}
\hline Type of gross lesion & Present study (\%) & Mardi et al (\%) & Mehendale et al (\%) \\
\hline Infarction & 56 & 16 & 41 \\
\hline Calcification & 10 & 28 & 35 \\
\hline Intervillous fibrin & 52 & 32 & - \\
\hline Subchorionic fibrin & 64 & 24 & 27 \\
\hline Retroplacental haematoma & 2 & 4 & 3 \\
\hline Normal & 16 & 40 & - \\
\hline
\end{tabular}

Table 6: Microscopic Examination

\begin{tabular}{|l|c|c|}
\hline Types of microscopic lesion & Out of 50 cases & \% \\
\hline Intervillous fibrin & 28 & 56 \\
\hline Syncytial Knots & & 0 \\
\hline$<30$ & 19 & 38 \\
\hline $30-50$ & 31 & 62 \\
\hline$>50$ & 24 & 48 \\
\hline Stromal fibrosis & 32 & 64 \\
\hline Infarction & & \\
\hline Basement membrane thickening & 20 & 40 \\
\hline $0-2 \%$ & 11 & 22 \\
\hline$>2 \%$ & 26 & 52 \\
\hline Cytotrophoblastic hyperplasia & & 56 \\
\hline Villous vascularity & 28 & 0 \\
\hline Normal & 0 & 44 \\
\hline Increased & 22 & 10 \\
\hline Decreased & 5 & \\
\hline Sclerosis of fetal stem vessels & & 40 \\
\hline Fibrinoid necrosis & 20 & 44 \\
\hline $0-5 \%$ & 22 & 16 \\
\hline $5-10 \%$ & 8 & 4 \\
\hline$>10 \%$ & 16 & \\
\hline Calcification & 2 & \\
\hline Mild & & \\
\hline Extensive & & \\
\hline Chronic villitis & & \\
\hline
\end{tabular}




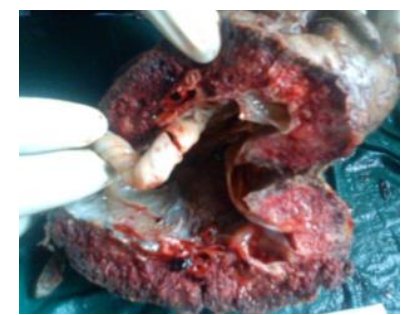

Fig.1: Gross specimen of small placenta showing extensive area of haemorrhage

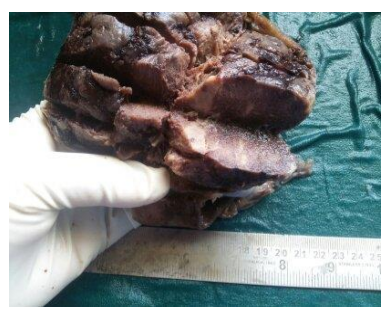

Fig. 2: Gross cut section of placenta showing an area of infarct

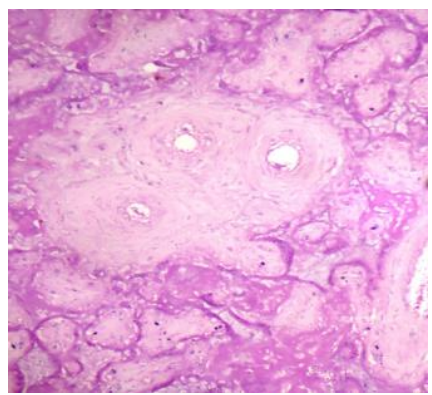

Fig. 3: Photomicrograph 10X(H \& E)- showing infarction necrosis of placenta

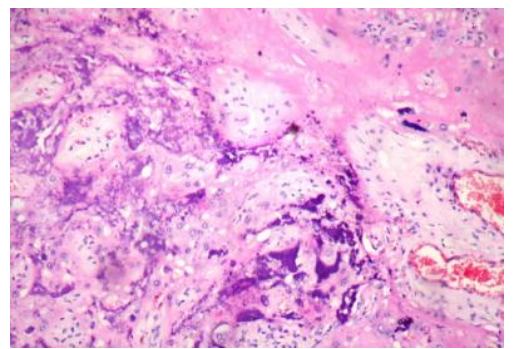

Fig. 4: Photomicrograph 10x(H \&E)- showing calcification in placenta

\section{Conclusion}

All the major gross and microscopic changes in the LBW placentae pointed towards reduced blood flow to the placenta resulting in chronic placental insufficiency. The end result of these events is growth retarded fetus. The severity of placental abnormalities expressed as cumulative number of placental lesions is a significant risk factor for LBWB. Morphological examination of the placenta is easy to perform, does not require an elaborate set up and can be performed routinely in a histopathological laboratory. Such an examination of the placenta conducted in the present study proved to be an useful adjuvant to the clinical examination in finding the pathogenetic mechanisms resulting in LBWB pregnancies and can be helpful in the planning and management of future pregnancies.

\section{Conflict of Interest: None.}

\section{References}

1. Bibbo M. placenta and low birth weight. In; Bibbo M, Grudman E, Kriesten WH. Perinatal pathology 1st edition New York: Springer-verlag Berlin Heidelberg;1979:pg157.

2. Davis BR, Casanueva E, Arroyo P. Placentas of small for date infants: A small controlled series from Mexico City, Mexico. Am J Obstet Gynecol 1984;149:731-736.

3. Mardi K, Sharma J. Histopathological evaluation of placenta in IUGR pregnancies. Indian J pathol Microbiol 2003;46:551-54.

4. Mehendale SS, Vasanthi L, Godbole PV. Placental Histopathology with IUGR. Indian J Obstet Gynecol 1988;38:406-409.

5. Bazaz G, Mirchandani JJ, Chitra S. Placenta in intrauterine growth retardation. Indian J Obstet Gynecol 1979;29:805-10.

6. Salafia CM, Vintziiloes AM, Silberman L. Placental pathology of idiopathic intrauterine growth retardation at term. Am J Perinatology 1992;9:179-84.

7. Bane AL, GillanJE, Masssive perivillous fibrinoid deposit causing recurrent placental failure. International J Obstet Gynecol 2003;100:292-295.

8. Mathew R, Aikat BM. Morphological study of placenta in abnormal pregnancy. Indian J pathol 1973;15:15-24.

9. Nigam J, Misra V, Singh P, Singh P, Chauhan S, Thakur B. Histopathological Study of Placentae in Low Birth Weight Babies in India. Ann Med Health Sci Res 2014;4(Suppl 2):S79-S83.

10. Hayward CE, Lean S, Sibley CP, Jones RL, Wareing M, Greenwood SL, et al. Placental Adaptation: What Can We Learn from Birthweight: Placental Weight Ratio? Front Physiol 2016;7:28.

11. Cibils LA. The placenta and newborn infants in hypertensive conditions. Am J Obstet Gynecol 1974;118(2):256-270.

12. Das B, Dutta D, Chakraborthy S, Nath P. Placental morphology in hypertensive of pregnancy and its correlations with fatal outcome. J Obstet Gynecol India 1996;40-46.

13. Panti AA, Ekele BA, Nwobodo EI, Yakubu A. The relationship between the weight of the placenta and birth weight of the neonate in a Nigerian Hospital. Niger Med J 2012;53:80-4.

14. Prabhjot K, Subhash K, Ashish S, Kuljit S. To Study Relationship of Umblical Cord Insertion with Fetal Outcome in PIH and Normotensive Pregnancies. Int $\mathbf{J}$ Pure App Biosci 2014;2:108-111.

15. Rath G, Garg K. Insertion of umbilical cord on the placenta in hypertensive mothers. J Anat Soc India 2000;49(2):149-52.

16. Amelia S, McLennan, Victoria XY, Cande VA, Cynthia GB, Russell SM. 277: Does abnormal placental cord insertion at anatomy ultrasound predict small-forgestational-age infants? Am J Obstet \& Gynecol 2017; 216:S170.

17. Macpherson TF and Fancy. What can we really tell from the placenta. Arch Pathol Lab Med 1991;115;672-79.

18. Murthy LS, Agarwal KN, Khanna S. Placental morphometric and morphological alteration in maternal 
under nutrition. Am J ObstetGynecol 1976;121(6):641645.

19. Prinz W, Schuhmann RA, Kalbfleisch W. Morphologic finding in the placenta in IUGR Zentralbl Gynakol 1983;105(5):279-86.

20. Rayburn W, Sander C, Compton A. Histological examination of placenta in growth retarded fetus. Am J Perinatol 1989;6:58-61.

21. Schindler RN. Importance of the placenta and cord in the defence of neurologically impaired infants claims. Arch Pathol Lab Med 1991;115:685-688.

22. Scott JM, Jordan JM. Placental insuffiency and small for date baby. Am J Obstet Gynecol 1972;113(6)823-832.

23. Salafia CM, Minor VK, pezzullo JC. Intrauterine growth restriction in infants of less than 32 weeks gestation: associated placental pathological features. Am J Obstet Gynecol 1995;173:1049-57.

24. Chandra P, Bal MS, Neeraja, Sunrinder K, Sethi PS. Histopathological changes in placenta in IUGR. Indian J Pathol Microbiol 2000;176-177.

25. Larysa A, Yao T, Richard G, Samuel O, John FDJ, Kweku. Maternal Demographic and Placental Risk Factors in Term Low Birth Weight in Ghana. J Preg Child Health 2017;4:325.

26. Mirchandani JJ, Bazaz MG, Chitra S. correlation of fetal outcome with some pathological changes in placenta. $\mathrm{J}$ obstet Gynaecol India 1979;29:1131-1138.

27. Dwason L, Lakshmi V, Salhan S, Zyerger B.

Histopathological changes in placenta in IUGR. Indian $\mathrm{J}$ Pathol Microbiol 1999;216-217.

28. Garcia AG. Placental morphology of LBW infants born at term. Contrib Obstet Gynecol 1982;9:100-12.

29. Altenmani AM, Gonzatti AR. Villitis of unknown etiology in placentas of pregnancies with hypertensive disorders and SFGA infants. Rev Assoc Med Bras 2003;49(1):67-71. 